\title{
ALBERT CAMUS ET LA RECHERCHE DU BONHEUR
}

\author{
MTCHEL SOULIÉ
}

\section{INTRODUCTION}

La mort, surtout lorsqu'elle frappe un homme encore jeune, fige la destinée en bouchant brutalement les possibles qui demeuraient ouverts. Pour un artiste, la coupure est encore plus marquée: il y trouve soudain un visage définitif et son oeuvre devient, d'un seul coup, parfaite. C'est un grand déchirement d'être mis ainsi devant la certitude qu'une voix s'est tue à jamais qui avait encore tant de choses à dire; mais c'est aussi une grande consolation que de trouver, dans l'oeuvre que laisse Camus, la présence d'une pensée pleine et forte et d'un art fait à la fois de lucidité et de poésie.

Camus pourtant est un des rares écrivains qui fasse mentir ce qu'il écrit lui-même dans ses "Cahiers" en février 1939: "De même que la mort d'un écrivain fait qu'on exagère l'importance de son oeuvre, la mort d'un individu fait qu'on surestime sa place parmi nous". Il a pris avec la mort et le recul du temps toute sa stature: elle est immense.

L'itinéraire spirituel de Camus n'avait pas atteint son terme: lorsque la mort l'interrompit tragiquement, il préparait un nouveau roman qui devait être l'oeuvre de sa maturité d'écrivain et il avait en vue un nouvel essai. Mais cet itinéraire était déjà riche en enseignements et, en un sens, achevé.

Car l'écrivain avait, sur les sujets majeurs qui l'avaient retenu (et qu'y avait-il pour lui de plus important que l' homme et son destin?), révélé déjà, dans une forme qui avait trouvé son équilibre et sa puissance, sa conception du monde et sa philosophie. Camus, certes, n'était pas un "homme à système", bien qu'il fût universitaire de formation. Sa pensée s'exprime aussi bien dans ses romans et dans ses pièces de 
théâtre que dans ses essais: même dans ces derniers, même dans un ouvrage comme "L'Homme révolté", elle n'a rien de figé ni de dogmatique. Elle reste toujours, malgré la solidité de sa structure logique, appuyée sur une expérience concrète et vraie et toujours le philosophe est inséparable de l'écrivain, de l'artiste, au sens plein du terme, du poète même.

\section{I - Le bonheur ou "le monde sans les hommes"}

Parler d'Albert Camus et de la recherche du bonheur, ce n'est donc pas définir abstraitement le concept de bonheur chez Camus, c'est suivre, à travers son oeuvre si diverse et pourtant si resserrée dans une unité profonde, qui est celle là même de sa personnalité, le chemin qu'il a parcouru jusq' au moment où la mort a brisé cette quête anxieuse. Camus écrit, en évoquant à l'âge d'homme, les images qui avaient marqué à jamais sa sensibilité d'adolescent et qui, tout au long de son oeuvre, éclairent son art:

"Oui, rien n'empêche de rêver, à l'heure même de l'exil, "puisque du moins je sais cela, de science certaine, qu'une "oeuvre d'homme n'est rien d'autre que ce long cheminement "pour retrouver par les détours de l'art les deux ou trois ima"ges simples et grandes sur lesquelles le coeur, une première "fois, s' est ouvert".

Le bonheur est précisément une de ces images et un des fils conducteurs qui permettent de suivre et de comprendre l'évolution de la pensée et de la vie d'Albert Camus. Ce mot ne cesse de retentir à travers presque toutes les pages de son oeuvre; il éclaire aussi sa vie; il commande même son action dans ce qu'elle a eu de plus décisif. N'a-t-il pas écrit, en parlant de la dernière guerre: "Nous autres, Français, nous "sommes entrés dans cette guerre, non pour le goût de la "conquête mais pour défendre justement une certaine idée de bonheur" - (Combat - 22 décembre 1944).

Le critique français, André Rousseaux, dans une de ses synthèses brillantes mais toujours approximatives, que permettent de simplifier l'infinie diversité du réel et de la réduire à quelques oppositions simples, pense que le bonheur, la place qu'on lui accorde et la valeur qu'on lui donne peuvent servir de ligne de partage entre deux familles d'esprit: d'un côté, les 
héros et les saints, que se sacrifient au salut, leur propre salut et le salut des autres; de l'autre, tous ceux qui suivent l'exigence du bonheur. Pour rattacher cette analyse à celle de Sainte-Beuve, on pourrait ranger les écrivains français en deux longs et glorieux cortèges: l'un prendrait la suite de Pascal, celui des héros et des saints; on y trouverait Vigny, Péguy et Claudel; l'autre suivrait Montaigne et Voltaire, celui de tous ceux qui revendiquent pour l'homme le droit d'être heureux sur cette terre. Camus est-il de cette lignée?

Il faut se garder de répondre trop vite à cette question: Camus n'est pas simple. Il se définit plus par une volonté toujours tendue de concilier, dans une unité vivante, les exigences contradictoires de l'homme que par des choix qui excluent totalement un des aspects de la nature humaine (cf. dans "Retour à Tipasa": "Il y a ainsi une volonté de vivre sans rien refuser de la vie qui est la vertu de l'homme à laquelle je tiens le plus en ce monde").

Le premier moment de cet itinéraire vers le bonheur, le plus important peut-être car il est comme la source de tous les autres et qu'il se retrouve épuré, mais encore vivant jusque dans la sérénité de l'homme fait, c'est le moment de l'adolescence à Alger; c'est celui que l'on pourrait appeler de moment du "monde sans les hommes". Camus est enraciné dans cette terre brûlée de soleil et chaude de passion, torturée aujourd'hui par la violence, pour laquelle il a toujours eu un attachement physique que rien n'a pu briser. Le drame de Camus l'Algérien n'est pas de mon propos: il est à beaucoup de points de vue le symbole même de cette guerre et de ses contradictions si dificiles à surmonter. Mais il intéresse l'ensemble de l'oeuvre de cet écrivain exemplaire et il est, au fond, le drame même de sa vie.

De sa jeunesse à Alger, où il fut un étudiant pauvre mais heureux, jeté dans les joies du corps et de la nature, Camus a até marqué jusqu'au plus profond de son être. Jamais il n' oubliera - comme le dit la citation que j'ai fait plus haut même dans son "exil" "les images simples et grandes" qui l'ont alors frappé. Dans cette même préface de "L'Envers et l'Endroit", il écrit avec lucidité, évoquant vingt ans plus tard son adolescence: "Je n'avais pas encore traversé les temps 
du vrai désespoir. Ces temps sont venus et ils on pu tout détruire en moi, sauf justement l'appétit désordonné de vivre" et, plus loin: "L'ardeur affamée qu'on sentira dans les Essais si souvent ne m'a jamais quitté et, pour finir, elle est la vie dans ce qu'elle a de pire et de meilleur". Qu'est-ce que cette ardeur, cet appétit? Ils ont une double face, à la fois complémentaire et contradictoire: le dégoût et le mépris pour les jeux vides et stériles de l'intelligence et même pour les constructions de la morale; la joie animale de communier avec la nature, l'immersion dans la vie élémentaire et dans les flots d'un "cosmos" qui enveloppe l'être humain et le fait retourner vers ses sources primitives.

La nature en Algérie et dans les pays méditerranéens où il voyagea (Italie-Espagne) est propre à l'exaltation du corps et le jeune homme se plongea, dès qu'il en eut l'âge, avant que la maladie ne vînt le frapper, dans cet océan de sensations physiques. Il a laissé le magnifique témoignage de cette communication panthéiste dans les courts essais poétiques de cette époque: "Noces - "L'Envers et l'Endroit", certains textes aussi recueillis plus tard dans "L'Eté". On retrouve aussi la marque brûlante de ce bonheur sensuel dans certains textes des "Cahiers" de cette époque et dans les pages de son roman: "La mort heureuse", terminé en 1937 mais jamais encore publié. Ce panthéisme sensuel, cet abandon aux forces élémentaires est un thème magnifique et la joie de vivre de Camus est alors confondue avec celle d'un corps bondissant dans les vagues de la mer, respirant le vent sur les plages ou jouissant du soleil et de sa présence au monde.

Les thèmes méditerranéens de Camus qui courrent tout au long de son oeuvre et que l'on retrouve en brèves notations dans les "Cahiers" qu'il tenait; la lumière, la chaleur, le soleil, le sourire des femmes, l'odeur des fleurs, la pureté du désert sont magnifiquement orchestrés dans une prose poétique qui est à la fois charnelle et lucide, comme dans presque toutes les pages des écrits de ce temps.

Voilà par exemple quelques extraits de "Noces" qui illustrent ce panthéisme et cette joie de vivre par les sens:

"Au bout de qualques pas, les absinthes nous prennent à la 
"gorge. Leur laine grise couvre les ruines à perte de vue. Leur "essence fermente sous la chaleur, et de la terre au soleil "monte sur toute l'étendue du monde un alcool généreux qui "fait vaciller le ciel. Nous marchons à la rencontre de l'amour "et du désir. Nous ne cherchons pas de leçons, ni l'amère phi"losophie qu'on demande à la grandeur. Hors du soleil, des "baisers et des parfums sauvages, tout nous parait futile. "Pour moi, je ne cherche pas à y être seul. J'y suis souvent "allé avec ceux que j'aimais et je lisais sur leurs traits le clair "sourire qu'y prenait le visage de l'amour. Ici je laisse à d'au"tres l'ordre et la mesure. C'est le grand libertinage de la na"ture et de la mer qui m'accapare tout entier. Dans ce ma"riage des ruines et du printemps, les ruines sont redevenues "pierres et perdant le poli imposé par l'homme sont rentrées "dans la nature".

Voici le vent: "Ce bain violent de soleil et de vent épui"sait toutes mes forces de vie. A peine en moi ce battement "d'ailes qui affleure, cette vie qui se plaint, cette faible ré"volte de l'esprit. Bientôt répandu aux quatre coins du monde, "oublieux, oublié de moi-même, je suis ce vent et dans le vent, "ces colonnes et ces arcs, ces dalles qui sentent chaud et ces "montagnes pâles autour de la ville déserte. Et jamais je n'ai "senti, si avant, à la fois mon détachement de moi-même et "ma présence au monde".

Voici les soirs d'Alger: "Ces courts instants où la journée "bascule dans la nuit, faut-il qu'ils soient peuplés de signes "et d'appels secrets pour qu'Alger en moi leur soit à ce point "liée? Quand" je suis quelque temps loin de ce pays, j'imagine "ses crépuscules comme des promesses de bonheur. Sur les "collines qui dominent la ville, il y a des chemins parmi les len"tisques et les oliviers. Et c'est vers eux qu'alors mon coeur "se retourne. J'y vois monter des gerbes d'oiseaux noirs sur "l'horizon vert. Dans le ciel, soudain vidé de son soleil, quel"que chose se détend. Tout un petit peuple de nuages rouges "s'étire jusqú'à se résorber dans l'air. Presque aussitôt après, "la première étoile apparaît qu'on voyait se former et se durcir dans l'épaisseur du ciel. Et puis, d'un coup, dévorante, la "nuit. Soirs fugitifs d'Alger, qu'ont-ils donc d'inégalable pour "délier tant de choses en moi? Cette douceur qu'ils me laissent 
"aux lèvres, je n'ai pas le temps de m'en lasser qu'elle dis"parait déjà dans la nuit. Est-ce le secret de sa persistence? "La tendresse de ce pays est bouleversante et furtive".

Voici Florence: "A Florence, je montais tout en haut du “jardin Boboli, jusqu'à une terrasse d'où l'on découvrait le "Monte Oliveto et les hauteurs de la ville jusqu'à l'horizon. "Sur chacune de ces collines, les oliviers étaient pâles comme "de petites fumées et dans le brouillard léger qu'ils faisaient "se détachaient les jets plus durs des cyprès, les plus proches "verts et ceux du lointain noirs. Dans le ciel dont on voyait "le bleu profond, de gros nuages mettaient des taches. Avec "la fin de l'après-midi, tombait une lumière argentée ou tout "devenait silence. Le sommet des collines était d'abord dans "les nuages. Mais une brise s'était levée dont je sentais le "souffle sur mon visage. Avec elle, et derrière les collines, les "nuages se séparèrent comme un rideau qui s'ouvre".

Voici Ibbiza, dans les Baléares: "Vers cinq heures, les "jeunes gens du pays se promènent sur deux rangs tout le "long de la jetée. Là se font les mariages et la vie tout en“tière. On ne peut s'empêcher de penser qu'il y a une certaine "grandeur à commencer ainsi sa vie devant le monde. Je m' "asseyais, encore tout chancelant du soleil de la journée, plein "d'églises blanches et de murs crayeux, de campagnes sèches "et d'oliviers hirsutes. Je buvais un orgeat douceâtre. Je re"gardais la courbe des collines qui se faisaient face. Elles "descendaient doucement vers la mer. Le soir devenait vert. "Sur la plus grande des collines, la dernière brise faisant tour"ner les ailes d'un moulin. Et, par un miracle naturel, tout "le monde baissait la voix. De sorte qu'il n'y avait plus que le "ciel et des mots chantants qui montaient vers lui, mais qu'on "percevait comme s'ils venaient de très loin. Dans ce court "instant de crépuscule, régnait quelque chose de fugace et "de mélancolique qui n'était pas sensible à un homme seu"lement, mais à un peuple tout entier. Pour moi, j'avais envie "d'aimer comme on a envie de pleurer. Il me semblait que "chaque heure de mon sommeil serait désormais volée à la "vie. . . c'est-à-dire au temps du désir sans objet. Comme dans "ces heures vibrantes du cabaret de Palma et du cloitre de 
"San Francisco, j'étais immobile et tendu, sans forces contre "cet immense élan qui voulait mettre le monde entre mes "mains".

Je me suis attardé sur ces citations pour beaucoup de raisons. La première c'est que cette prose poétique, qui surgit plus tard seulement par places dans l'oeuvre de Camus, est très belle et très émouvante: elle relie Camus à la terre natale et elle enracine sa philisophie dans un sol vivant qui l'empêchera de jamais se perdre dans l'abstraction d'une pensée sèche. Ce n'est pas un mot en l'air que celui que j'ai cité: les images de son adolescence le marquent toute la vie et, comme il le dit encore dans la préface de "L'Envers et l'Endroit": ma source est dans ce monde de pauvreté et de lumière où j'ai longtemps vécu". Dans presque tous ses ouvrages en effet, on retrouve l'appel, tantôt puissant, tantôt discret, souvent transformé et épuré, de la vie physique, du contact vivifiant et enrichissant avec les forces élémentaires de la nature. Sans parler de la description de la baignade dans "la Peste", lisez dans le recueil intitulé "L'Exil et le Royaume" (un titre qui plonge aussi ses racines dans la séparation avec la terre natale), la fin de la nouvelle "La femme adultère" ou encore "La pierre qui pousse". On y retrouve dans ces textes de l'âge d'homme, le même élan cosmique qui poussait le jeune Camus vers les plaisirs simples et pourtant infiniment vastes du corps. Toujours, même après la longue évolution qui aboutira à sa dernière conception de la vie, Camus conservera, comme la source vivante du bonheur, l' ouverture sensible sur le monde qu'il découvrait avec émerveillement lorsqu'il avait vingt ans.

Rien de plus significatif que le texte intitulé: "Retour à Tipasa" écrit en 1952. Tipasa est le lieu des "Noces" décrites dans l'Essai qui porte ce titre: Camus y retourne dix-huit ans après, en 1952, et cette espèce de "Temps retrouvé" est aussi une méditation sur la joie de vivre et ses rapports avec le bonheur. Ce qui ressort de ce pélerinage aux sources, dans lequel Camus retrouve le style plein et sensuel de ses vingt ans, c'est l'inépuisable exigence de vie qui, épurée de sa violence primitive et transfigurée, fonde l'action tout entière de Camus et l'alimente jusque dans ses prolongements les plus 
généreux et les plus éloignés de cette fusion panthéiste avec la nature.

"Pour empêcher que la justice se racornisse, beau fruit "orange qui ne contient plus qu'une pulpe amère et sèche, je "redécouvrais à Tipasa qu'il fallait garder intactes en soi une "fraicheur, une source de joie, aimer le jour qui échappe à "l'injustice, et retourner au combat avec cette lumière con“quise. Je retrouvais ici l'ancienne beauté, un ciel jeune, et "je mesurais ma chance, comprenant enfin que dans les pires "années de notre folie le souvenir de ce ciel ne m'avait jamais "quitté. C'était lui qui pour finir m'avait empêché de déses"pérer. Au milieu de l'hiver, j'apprenais enfin qu'il y avait en "moi un été invincible".

Je voudrais remarquer ici combien le thème de la lumière est à la fois riche et important chez Camus: de purement matériel, il devient le symbole de la pensée droite et juste, la "pensée de midi" de l'Homme Révolté" et finalement de toute une attitude morale face au monde. Il n'y a rien là d'original; mais ce qui est puissant, ce sont les racines charnelles de ce thème, qui tiennent au plus profond de la conscience et de la sensibilité de Camus. Il en prend une valeur poétique qui le renouvelle.

La deuxième raison de l'importance de ce premier moment de la recherche du bonheur chez Camus, c'est pourraiton dire, son aspect négatif. Ce cantique de la joie de vivre se double d'une philosophie nihiliste: il n'ya ni Dieu, ni vie éternelle; la mort est certaine et elle est le mal absolu puisque la vie corporelle est le seul bien incontestable: la seule joie possible, c'est l'acceptation lucide par l'homme de sa condition, c'est-à-dire la jouissance de son être. L'adhérence du corps à l'univers fait sombrer l'esprit et l'espérance même dans un néant où seul surnage la certitude d'exister. (C'est en ce sens, mais en ce sens seulement, que Camus a pu passer pour existentialiste: on voit tout de suite que cette attitude est limitée à un moment de son itinéraire intellectuel; bien plus, elle est simplement une attitude de vie, nullement une philosophie systématique comme chez les existentialistes, qu'ils soient chrétiens ou athées; dès que Camus réfléchit sur cet engagement, il le dépasse et cesse d'être existentialiste). Sur ces 
thèmes. Camus multiplie les formules éclatantes et poétiques mais au fond sans ouverture: "J'aime cette vie avec abandon "et veux en parler avec liberté: elle me donne l'orgueil de ma "condition d'homme. Pourtant on me l'a dit souvent: il n'y "a pas de quoi être fier. Si, il y a de quoi: ce soleil, cette mer, "mon coeur bondissant de jeunesse, mon corps au goût de "sel et l'immense décor où la tendresse et le génie se rencon"trent dans le jaune et le bleu".

Cette ivresse des sens aboutit même parfois à une voIonté de rejeter totalement l'esprit. Tandis que Barrès montait sur la colline de Sion, pour y hanter "les lieux où souffle l'esprit", Camus se plait sur la colline de Djemila "où meurt l'esprit pour que naisse une vérité qui est sa négation même". "Bientôt oublieux, oublié de moi même, je sens ce vent et, dans le vent, ces colonnes et cet arc, ces dalles qui sentent chaud et ces montagnes pâles autor de la ville déserte. Et jamais je n'ai senti si avant, à la fois mon détachement de moi-même et ma présence au monde".

Dans ses "Cahiers", en janvier 1936, il exprime cet abandon au monde où il se retrouve en se perdant. Il nuance sa joie presque dyonisiaque du sentiment de la fuite du temps et de l'angoisse de la mort. Mais cette angoisse elle-même donne plus de prix au bonheur: "Etre ce rayon de soleil où "ma cigarette se consume, cette douceur et cette passion discrète qui respire dans l'air. Si j'essaie de m'atteindre, c'est "tout au fond de cette lumière. Et si je tente de comprendre "et de savourer cette délicate saveur qui livre le secret du "monde, c'est moi-même que je trouve au fond de l'univers.

"Si une angoisse encore m'étreint, c'est de sentir cet "impitoyable instant glisser entre mes doigts comme les perles "du mercure. Je suis heureux dans ce monde car mon royau"me est de ce monde".

Devant les collines de Florence, dans cette méditation, dont j'ai cité déjà une page, Camus découvre que "le monde "est beau et qu'hors de lui, il n'y a point de salut". La vérité que lui enseigne ce paysage “c'est que l'esprit n'est rien, ni "le coeur même. Et que la pierre chauffée par le soleil ou le "cyprès que le ciel découvert agrandit, limitent le seul univers "où avoir raison prend un sens: la nature sans les hommes". 
Cet hymne à la vie se sépare même parfois brutalement, avec une rudesse qui n'est sans doute qu'impatience de jeune homme, de toute consciente philosophique ou même de toute communion artistique (alors que ce dernier élément est toujours présent chez Giono, chez Montherlant, chez Gide, sans parler bien entendu du Rousseau des "Rêveries"), Camus semble alors vouloir retourner à l'abêtissement d'une vie purement sensuelle: "Puis-je, écrit-il dans une note de "Noces", me donner le ridicule de dire que je n'aime pas la façon dont Gide exalte le corps? Il lui demande de retenir son désir pour le rendre plus aigü. Ainsi se rapproche-t-il de ceux que dans l'argot des maisons publiques on appelle les compliqués ou les cérebraux. Le christianisme aussi veut suspendre le désir. Mais, plus naturel, il y voit une mortification. "Mon camarade "Vincent, qui est tonnelier et champion de brasse junior, a "une vue des choses encore plus claire. Il boit quand il a soif; "s'il désire une femme cherche à coucher avec, et l'épouserait "s'il l'aimait (ça n'est pas encore arrivé). Ensuite, il dit tou"jours: "Ça va mieux-ce qui résume avec vigueur l'apologie "qu'on pourrait faire de la satiété".

Tel est, très rarement, exceptionnellement même l'aboutissement extrême de ce mouvement qui représente alors un défi d'adolescent à l'intelligence et à la morale traditionnelle. La joie de vivre peut dégénérer en effet en dégradation, comme elle peut devenir la source vivante d'une vertu de vivre. Camus a été sauvé de cette dégradation, non par la morale formelle, non même par le souci de préserver l'esprit, non plus par la croyance en Dieu, mais par une double exigence: de dignité personnelle d'une part et d'amour pour les autres hommes d'autre part. Elle lui a permis de ne jamais tomber dans un "hédonisme" grossier. Car au moment même où il décrivait la sagesse comme le "singulier instant où la spiritualité répudie la morale, où le bonheur naît de l'absense, d'espoir, où l'esprit trouve sa raison dans le corps, en ce temps de l'innocence où, comme il le dit lui-même, il ignorait que la morale existât, il pressentait déjà que cette communion instantanée avec le monde était trompeuse, insuffisante en tout cas. $N$ ' écrit-il pas, dans ses "Cahiers", en 1937, dans l'ivresse de sa communion avec la nature, les lignes suivantes riches d'une 
promesse de dépassement du plaisir sensuel et d'un besoin d'une ascèse d'un gouvernement du corps.

C'est dans un dénuement lucide qu'il trouve le bonheur: "Consentir au monde et en jouir, mais seulement dans le dénuement. Je ne serais pas digne d'aimer la nudité des plages si ne savais demeurer nu devant moi-même. Pour la première fois, le sens du mot bonheur ne me parait pas équivoque. Il est un peu le contraire de ce qu'on entend par l'ordinaire: "Je suis heureux".

Il existe aussi, dès cette période, une autre ouverture dans le monde de la sensualité de Camus: c'est la conscience de l'existence des autres, de la grande misère des hommes et c'est aussi la volonté inflexible de ne pas s'incliner devant le mal. La révolte est déjà au coeur de cette acceptation du monde et, étroitement uni à elle, l'amour.

Lisons ce qu'il écrit dans les "Cahiers", en septembre 1937, en méditation dans le cloître des morts à la Santissime Annunziata à Florence: "Si j'avais à écrire ici un livre de morale, il "y aurait 100 pages et 99 seraient blanches. Sur la dernière j'écrirais: Je ne connais qu'un seul devoir et c'est celui d' aimer. Et pour le reste, je dis non, je dis non de toutes mes "forces. Les dalles (du cloître) disent que c'est inutile... Mais "je ne vois pas ce que l'inutilité ôte à ma révolte et je sens "bien ce qu'elle lui ajoute".

Retrouvons-en l'écho dans la page de "Noces", où il exalte l'enseignement de son voyage à Florence: "J'admirais, j'admi"re ce lien qui, au monde, unit l'homme, ce double reflet dans "lequel mon coeur peut intervenir et dicter son bonheur jusqu' “à une limite précise où le monde peut alors l'achever ou le "détruire. Florence! Un des seuls lieux d'Europe où j'ai compris qu'au coeur de ma révolte dormait un consentement. Dans "mon ciel mêlé de larmes et de soleil, j'apprenais à consentir "à la terre et à brûler dans la flamme sombre ses fêtes. "J'éprouvais... mais quel mot? Quelle démesure? Comment "consacrer l'accord de l'amour et de la révolte? La terre! Dans "ce grand temple déserté par les dieux, toutes mes idoles "ont des pieds d'argile". 


\section{II — De "l'absurde" à l'amour des hommes par la révolte.}

Malgré cete promesse, le passage de la pure joie de vivre au bonheur se fera après la traversée d'un désert moral et intellectuel, comme d'ailleurs le pressentait l'auteur de "Noces", qui voulait s'enivrer des plaisirs physiques pour ne pas se laisser absorver par le tragique de la condition humaine. Le choc qui déterminera la rupture avec cet appétit de vivre au rythme des jouissances sensuelles fut la guerre et ses exigences.

Dans "Retour à Tipasa", il marque bien les étapes de son itinéraire. "Elevé d'abord dans le spectacle de la beauté qui était ma seule richesse, j'avais commencé par la plénitude. Ensuite étaient venus les barbelés je veux dire les tyrannies, la guerre, les polices, le temps de la révolte. Il avait fallu se mettre en règle avec la nuit. Comment oublier que le romancier de "l'Etranger" et le philosophe du "Mythe de Sisyphe" était aussi le résistant qui avait dit "non" au mensonge, à la défaite, à l'injustice? Le spectacle de la misère de la condition humaine, dévoilée dans sa nudité par la guerre, la certitude de l'impasse où aboutissait la pure joie sensuelle, la découverte que, comme le dit Caligula: "les hommes meurent et ne sont pas heureux", toutes ces évidences que Camus soupçonnait (Caligula fut écrit en 1938) et contre lesquelles il cherchait remède dans une communion avec la nature aboutissent à une prise de conscience qui se manifeste par la philosophie de "l'absurde".

Ce moment de l'absurde, on en a beaucoup parlé: il joue un rôle important dans l'oeuvre et dans l'itinéraire de Camus, mais ce n'est qu'un moment, un moment dialectique pour être plus précis, qui permet une marche en avant, un dépassement vers d'autres certitudes. La vue lucide de l'absurdité de la condition humaine a été nécessaire à Camus pour fonder d'autres valeurs. Dans la préface au "Mythe de Sisyphe", Camus écrit: "On peut dire qu'il y a du provisoire dans "mon commentaire; on ne saurait préjuger de la po"sition qu'il engage". Dans les premières pages de "L'Homme Révolté", il compare cette déniarche au "doute méthodique" de Descartes. Rien donc de plus faux et de plus stupide de figer Camus dans sa philosophie de l'absurde: elle est une 
étape dans un progrès qui débouche sur des horizons d'espoir et de confiance dans l'homme.

Dans cette marche dialectique, Camus s'exprime à la fois par des essais philosophiques et par des ouvrages d'imagination: soit pièces de théâtre, comme "le Malentedu" et "Caligula", soit romans. La marche est ouverte par une espèce de tétralogie où le monde "absurde" est cerné et mis en lumière: deux pièces, "Caligula" et le "Malentedu", un roman: "l'Etranger", un essai: "le Mythe de Sisyphe". Elle trouve son achèvement dans l'affirmation d'un humanisme rénové qui s'exprime d'une part dès 1947 dans le chef d'oeuvre de Camus: "la Peste" et, d'autre part, en 1951, dans un ouvrage théorique où se trouvent rassemblés les éléments de cette nouvelle philosophie: "L'Homme Révolté".

Le nihilisme de l'absurde éclate dans cette extraordinaire pièce qu'est "Caligula", où il est décrit jusqu'à ses plus extrêmes conséquences. Comme Gérard Philippe y était beau dans ce rôle de prince de l'enfer, assoiffé d'absolu et poussant jusqu'aux crimes les plus odieux l'amour désespéré qu'il porte aux hommes! "Caligula" est la tragédie de la lucidité devant l'insoutenable misère de la condition humaine et de la prise de conscience de l'absurdité de la vie; il est aussi la tragédie de l'échec d'une liberté sans frontières, de l'impasse où l'on s'enferme si, en se privant de Dieu, en croyant que "tout est permis" s'il n'existe pas, comme le disait Yvan Karamazov dans Dostoïewsky, on refuse aussi toute morale humaine. "Ma liberté n'est pas la bonne", dit Caligula, en se livrant aux épées des patriciens. Mais pourtant l'élan qui le soutient, la volonté de ne pas accepter le monde absurde et de trouver de quoi combler cette immense soif humaine est la promesse, lorsqu'ils seront dirigés dans la droite voie, d'une ouverture dans le cercle infernal où l'homme s'agite. Caligula veut conquérir "la lune"; s'il disciplinait sa révolte, s'il atteignait à cette 'mesure de midi", où la sagesse méditerranéenne se concentre, sans perdre la ferveur qui le pousse à l'action, il serait sans doute un empereur bienfaisant et un homme heureux. Mais cette leçon n'est encore qu'une petite lueur tremblotante dans le monde glacé, terrifiant et noir où se débat l'Empereur dément. 
Le nihilisme total ou presque éclate aussi dans "l'Etranger", ce roman qui, en 1942, fit connaître Camus à tous les Français qui, dans ces années sombres, ne désespéraient pas de notre jeune littérature. Quelle rupture dans le style-sauf pour quelques pages rayonnantes sur les plaisirs sensuels de la vie à Alger-avec la poésie des premiers Essais: Camus se range, par la sécheresse de ses descriptions, la précision de ses analyses, la justesse de ses notations psychologiques, dans la lignée des moralistes français. Dans une étude copieuse et profonde, Sartre, dès la publication du livre, souligna que "I'Etranger", par son style coupant et son pessimisme intellectuel, est proche de "l'Ingénu" de Voltaire. Certaines pages peuvent, en effet, faire penser à la sécheresse désespérante et destructrice de l'auteur de "Candide". Mais au-delà des petites phrases précises et volontairement nues, derrière le terrible dépouillement du style et du fond, se dessine toute une conception du monde bien éloignée, au moins dans ses prolongements, de l'hédonisme sans illusion de Voltaire: la leçon de "l'Etranger", Camus la tirera, un an plus tard, dans "le Mythe de Sisyphe" où il développera la philosophie qui sous-tend le récit.

Meursault, le héros de "l'Etranger", ce demiraté qui subit la vie et le monde, qui commet un meurtre sans en avoir l' intention et sans en concevoir de remords, est proche parent de ce Sisyphe que Camus, dans son "Essai" prend comme symbole de la condition humaine. Mais ce n'est qu'un des aspects du Sisyphe humain: le sentiment de l'absurde qui, dans le récit, apparaissait comme une donnée immédiate de la conscience, devient, au terme d'une analyse fouillée, le point de départ de toute une philosophie. Sisyphe roule sa pierre, dans un monde dont il a reconnu l'absurdité et l'insignifiance radicales: mais il accomplit cette tâche avec une lucidité totale, les yeux ouverts et le coeur fier; il jette un défi au monde qui l'accable. L'éveil de la conscience de l'absurde est déjà un commandement de victoire. "Lever, tramway, quatre heures de travail, repas, sommeil et lundi, mardi, mercredi, jeudi, vendredi et samedi sur le même rythme, cette route se suit aisément la plupart du temps. Un jour seulement, le "pourquoi s'élève et tout commence dans cette lassitude teintée d'étonnement". Ce commencement, cet éveil, certes, aboutit 
à dresser autour de l'homme un monde qui n'a pas de sens et à faire peser sur lui le poids d'une absurdité qui l'accable. Mais Camus s'engage alors dans une démarche qui fait penser à celle de Pascal: "Quand l'univers l'écraserait, l'homme serait encore plus noble que ce qui le tue, parce qu'il sait qu'il meurt et l'avantage que l'univers a sur lui, l'univers n'en sait rien".

Désormais, pour Camus, c'est cette conscience éveillée qui va porter tout l'espoir d'un avenir humain, sans que l'on vienne l'assister. Dans le dialogue entre le monde qui l'accable de sa masse incompréhénsible et l'homme, la prise de conscience est le début d'une marche qui aboutira à la victoire des valeurs humaines et qui permettra de fonder, sur des bases indestructibles puisqu'elles ont été bâties sur les ruines de toutes les anciennes illusions, la morale que Camus poursuit et qu'il entrevoyait confusément au delà de l'ivresse sensuelle de la communion avec la nature.

Meursault, à la fin de "1'Etranger", après s'être revolté contre l'injustice et l'absurdité de son destin -il a été condamné à mort pour un crime qu'il a commis sans y penser et sans en être responsable- sent un immense apaisement l'envahir; il accepte cette absurdité et cette injustice, il s'ouvre à "la tendre indifférence du monde" qui lui donne une sorte de bonheur. Et nous retrouvons ici le fil conducteur de la recherche du bonheur qui court à travers toute l'oeuvre de Camus: par delà le désespoir et l'absurdité du monde, en dehors cette fois des plaisirs purement physiques, l'exigence du bonheur de l' homme doit être satisfaite; c'est sur cette pierre que la morale pourra s'édifier. Mais le bonheur de Meursault est encore passif; il n'est guère que l'acceptation, après la révolte inutile, d'un destin dont l'absurdité même fait sa grandeur; il est la face négative d'un stoïcisme qui peut déboucher sur le fatalisme, tentation éternelle des peuples méditerranéens, gorgés de lumière et ivres d'engourdissement.

Sisyphe, lui, bâtit son bonheur sur une réalité plus solide et plus vivante; son éternel supplice marque une étape vers la délivrance. Certes, l'univers est vide, certes les dieux sont morts; certes le destin de l'homme est absurde. Mais, dans l'épuisante marche de Sisyphe, qui roule son rocher en sachant qu'il retombera sans cesse, l'effort humain, la lumière de la 
conscience contiennent déjà la certitude du salut, puisqu'ils affirment, face à une réalité incompréhensible et hostile, l' éternelle volonté de l'homme de n'abdiquer ni la dignité de sa pensée, ni l'efficacité de son action, ni enfin son exigence du bonheur. "Sisyphe enseigne la fidélité supérieure, qui nie les dieux et soulève les rochers. Lui aussi juge que tout est bien. Cet univers, désormais sans maitre ne lui paraît ni stérile, ni futile. La lutte vers les sommets suffit à remplir un coeur d'homme. Il faut imaginer Sisyphe heureux".

Ainsi se trouve résolue l'énigme, comme Albert Camus le dit lui-même, dans le court essai qui porte ce titre. Car ce philosophe de l'absurde, cet amant des plaisirs physiques, ce cynique volontaire, au moment même où il décrivait un monde irrationnel et vide, se lançait dans une action dangereuse, épuisante et désintéressée, en participant activement à la Résistance.

Ces deux attitudes ne sont pas contradictoires mais complémentaires: le nihilisme n'est qu'un point d'appui pour le dépasser; Camus ne s'y est jamais attardé, encore moins complu. Et ce qui l'a poussé en avant, ce qui l'a aidé dans le dépassement difficile d'une étape accablante, c'est précisément, comme je le disais, à la fois l'exigence invincible de bonheur qu'il sentait en lui et le souvenir de la lumière et de l'équilibre qu'il avait connus du temps de son adolescence: "Au plus noir de notre nihilisme, écrit-il en 1950, j'ai cherché seulement des raisons de dépasser ce nihilisme. Et non d'ailleurs par vertu, ni par une rare élévation de l'âme, mais par fidélité instinctive à une lumière où je suis né et où depuis des millénaires, les hommes ont appris à saluer la vie jusque dans la souffrance. Au centre de notre oeuvre, fût-elle noire, rayonne un soleil inépuisable".

Le mythe de Sisyphe n'est pourtant que la promesse de l'action héroïque; il n'en est pas l'accomplissement. Pour expliquer l'épanouissement de la morale de Camus en un humanisme rayonnant, pour aller de "l'Etranger" à la "Peste" et du "Mythe de Sisyphe" à "l'Homme Révolté", l'expérience de la Résistande est l'explication la plus forte. Au milieu même des horreurs d'une guerre inexpiable, dans un moment où la civilisation, dans ce qu'elle a à la fois de plus rare et de 
plus précieux, est menacée, Camus découvre la valeur de la fraternité. Cet individualiste voit s'ouvrir toutes grandes les portes d'un monde qu'il avait à peine aperçu jusqu'ici, où la sympathie et l'amour triomphent de l'hostilité d'une nature absurde et écartent aussi les tentations de l'homme seul.

Cete transformation, cette conversion même ne vont pas sans obstacles ni reculs. En 1936, en plein dans l'épanouissement du bonheur sans les hommes, il pousse dans les "Cahiers" ce cri, où l'on peut voir l'égoïsme superbe de l'individualisme: "Vais-je me demander si quelque chose meurt et si les hommes souffrent, puisque tout est écrit dans cette fenêtre où le ciel déverse sa plénitude. Instant d'adorable silence. Les hommes se sont tus. Je ne sais pas ce que je pourrais souhaiter de mieux que cete continuelle présense de moi-même à moi-même".

Dix-huit mois plus tard, je l'ai dit, son voyage à Florence lui ouvre d'autres perspectives: la révolte et l'amour, indissolublement jointes, font craquer l'univers parfait de ce bonheur égoïste. Mais cet éclatement n'est pas complet ni définitif. Malgré le premier choc de la guerre, la conversion ne se fera pas vite. L'individualisme résiste et essaie d'échapper à la prise de la fraternité. La lecture des "Cahiers" est significative à cet égard: on y relève des mouvements de recul devant l'action nécessaire, et l'amour des hommes, qu'il accepte pourtant mais sans joie. Il n'a pas encore retrouvé, dans ce nouvel état, le bonheur que l'égoïsme sensuel lui avait donné.

Il écrit, en juin 1938: "La seule fraternité maintenant possible, la seule qu'on nous offre et qu'on nous permette, c'est la sordide et gluante fraternité devant la mort militaire". En septembre 1938, alors qu'il cherche en vain à s'engager, c'est la résignation à l'inévitable et le sens de l'honneur qui inspirent son geste, non l'allégresse de l'acte spontané et la poussée vers le bonheur: "Les tours d'ivoire sont tombées écrit-il; la complaisance est interdite pour soi-même et pour les autres". Mais il se fait un serment: "Jurer de n'accomplir, dans la moins noble des tâches, que les plus nobles des gestes. Et le fond de la noblesse (la vraie, celle du coeur) c' est le mépris, le courage et l'indifférence profonde.

A Paris, au printemps de 1940, exilé de son Algérie (le 
thème de l'exil, si souvent repris par lui, correspond à une blessure profonde de sa sensibilité) lassé, écoeuré, près du désespoir, il perd, au moins pour un temps, la conscience de la révolte. Il retombe, en ces heures de doute, dans la tentation de l'individualisme (cf. les Cahiers - mars 1940).

De plus en plus, devant le monde des hommes, la seule réaction est l'individualisme. L'homme est à lui seul sa propre fin. Tout ce qu'on tente pour le bien de tous finit par l'échec. Même si l'on veut toutefois le tenter, il est convenable de le faire avec le mépris voulu.

Et en 1941, de retour en Algérie, plus calme, plus proche d'un nouvel équilibre sous le ciel de son adolescence, il jette ce cri où éclatent à la fois une promesse de fraternité et un dégoût "du monde avec les hommes". "Il faut payer et se salir à l'abjecte souffrance humaine. Le sale, refoussant et visqueux univers de la douleur".

C'est dans les années 1942 et 43 que Camus bascule définitivement du côté de la fraternité et qu'il découvre, comme Vigny avec lequel il a beaucoup de points communs, "la majesté des souffrances humaines" dont il voyait jusqu'ici surtout l'horreur. Tout au long de ce petit livre écrit au milieu du tumulte de la guerre, que constituent les "Lettres à un ami allemand", on sent qu'une étape de l'itinéraire de Camus est franchie. Il passe de la solitude à la solidarité, ces deux pôles de l'attitude humaine, qu'il évoquera, curieusement d'ailleurs, par une espèce de jeu de mots ambigüe, dans une nouvelle de son recueil: "L'exil et le royaume", lorsque le peintre Jonas, à demi-fou et abandonné de tous ses amis, écrit, sur sa dernière toile blanche, un mot dans lequel sa femme ne sait pas reconnaître "solitaire" ou "solidaire".

L'ambition ultime de Camus, c'est de réconcilier la solitude et la solidarité, l'homme et la société, l'art et l'action, dans une synthèse où l'individu s'épanouit dans la personne.

"La Peste", admirable roman que je tiens pour le chef d'oeuvre de Camus, est précisément l'expression, dans une forme romanesque, de la découverte de la solidarité humaine. Certes, il y avait déjà, même dans l'ivresse de "la nature sans les hommes" ou dans la vue lucide de "l'absurdité du monde", 
le germe de cet amour pour la créature qui fonde, à partir de "la Peste" la morale de Camus.

Dans "L'Etranger", Meursault découvre dans un banal patron de bistrot, un coeur fraternel: "C'est la première fois de ma vie que j'ai eu envie d'embrasser un homme".

Dans "Le mythe de Sisyphe", une ouverture sur l'univers des hommes écarte parfois les ténèbres du monde hostile; mais Camus le dit expressément, c'est un luxe: "Il n'y a qu'un luxe et c'est celui des relations humaines... Visages tendus, fraternité menacée, amitié si forte et si pudique des hommes entre eux, ce sont les vraies richesses, puisqu'elles sont périssables".

"J'aime la majesté des souffrances humaines". Ce cri de Vigny, avec lequel Camus a une parenté de conscience évidente, l'auteur de "la Peste" a voulu lui donner toute sa valeur. Dans Oran isolé du monde et voué à la peste - le choix de ce cadre confirme d'une part l'influence profonde de la terre algérienne sur Camus, d'autre part l'importance des années de guerre et de résistance dans son évolution, puisqu' Oran symbolise, au moins à une première analyse, la France de l'occupation- le docteur Rieux, Tarrou, Rambert, le père Paneloux aussi, se trouvent confrontés au malheur et à la souffrance. Ils ne se contentent pas de l'aimer, au sens fort où l'entendait Vigny; ils luttent contre elle et, dans cette lutte, ils trouvent tous, chacun à leur manière, la chaleur d'une communion avec les hommes. C'est cette sympathie, fondée sur l'amour, qui inspire leur action et leur lutte et qui brise, pour chacun d'eux, l'isolement où les enfermait une vie qui ignorait et le malheur des autres et le sacrifice.

Cette morale du sacrifice et de l'amour repose aussi sur la révolte. Avant même de systématiser, dans "l'Homme Révolté" une théorie de la morale fondée sur le refus du monde, de son absurdité, du mal et de l'injustice qu'il enveloppe, avant d'avoir exploré tous les chemins qu'ouvre cette volonté jaillie du plus profond de la conscience de ne pas accepter la malédiction du destin de l'homme, Camus, dans "la Peste", dans "les remarques sur la Révolte" dans "Les lettres à un ami allemand", avait déjà mis l'accent sur cette source intarissable d'action liée à une exigence irrésistible de l'homme. 
"Je refuserai jusqu'à la mort d'aimer cette création où des enfants sont torturés". Cette affirmation passionnée et tranquille de Rieux au Père Paneloux, qui prêche la résignation chrétienne, est l'expression de la volonté humaine de ne jamais accepter le mal; elle est aussi l'abandon de toute mystique qui justifie, par un au-delà insaisissable, l'injustice présente du monde.

La morale de "L'Homme Révolté", celle de "La Peste" (ce sont les mêmes) remplace-t-elle la morale du bonheur qui, aussi bien chez Camus de "Noces" que chez l'auteur du "Mythe de Sisyphe", était la valeur capitale par une morale du sacrifice? Elle introduit une nouvelle dimension dans l'univers de Camus; elle l'élargit en l'humanisant; mais elle n'en détruit pas l'essentiel. Dans le dépassement que représente, par rapport aux positions premières, la conception humaine du monde, les exigences primitives sont maintenues: rien $n$ ' est renié de la joie de vivre qui illuminait les jours heureux de l'adolescence de Camus; elle fait partie maintenant d'un nouvel édifice moral auquel elle donne sa lumière et qui, à son tour, lui restitue un équilibre qui lui faisait défaut.

Mais le dialogue entre le bonheur et le sacrifice est perpétuellement menacé; pour le maintenir dans sa pureté, pour ne pas tomber, soit dans une morale du sacrifice qui ignore l'exigence $\mathrm{du}$ bonheur, soit dans une morale du plaisir qui méprise l'appel de la justice, un effort continue de la conscience est nécessaire qui est la vie même de l'homme et qui fait la grandeur de sa condition. Dans "Les Lettres à un ami allemand", Camus demande "un juste équilibre entre le sacrifice et le goût du bonheur"; dans "Remarque sur la Révolte", il insiste plus clairement encore sur "le balancement perpétuel qui mène l'homme révolté de la volonté du sacrifice à l'exigence généreuse du bonheur".

Dans "La Peste", ce dialogue prend chair et vie en s'incarnant dans des personnages qui aiment et qui souffrent. Rambert, un journaliste qui se trouve enfermé par hasard dans Oran, cherche à quitter la ville pour aller rejoindre celle qu'il aime. Rieux, le docteur, qui organise la lutte contre la peste, qui l'a fait entrer dans les équipes sanitaires, a choisi le sacrifice; mais il ne blame pas Rambert: "Peut-être en effet 
suis-je dans mon tort en choisissant l'amour" 's'écrie celui-ci). Rieux lui fit face: "Non, dit-il, avec force, vous n'êtes pas dans votre tort". Rambert, au dernier moment, reste dans la ville. Rieux, une fois de plus affirme la vérité du bonheur: "Il se redressa et dit, d'une voix ferme, que cela était stupide et qu'il n'y avait pas de honte à préférer le bonheur - Oui, dit-Rambert, mais il peut y avoir de la honte à être heureux tout seul".

Tarrou, qui lui aussi a choisi le sacrifice, voudrait poser le problème en termes brutaux. "Tarrou fit remarquer que si Rambert voulait partager le malheur des hommes, il n'aurait plus le temps pour le bonheur. Il fallait choisir". Mais Rambert refuse le choix. Il reste, mais il ne veut pas abdiquer sa soif de bonheur et il interroge, anxieux: "Avez-vous donc choisi, vous, et renoncé au bonheur?- Ni Tarrou, ni Rieux ne répondirent encore... Et Rambert de nouveau pose sa dernière question avec plus de force encore. Et seul Rieux se tourne vers lui... Il reprit en regardant devant lui: "Rien au monde ne vaut qu'on se détourne de ce qu'on aime. Et pourtant je m'en détourne moi aussi sans que je puisse savoir pourquoi". (la femme de Rieux, malade, est hors d'Oran. Elle mourra sans qu'il la revoie).

Cette exigence mystérieuse, qui termine par une décision sans appel le dialogue du bonheur et du sacrifice, s'impose à l'homme, non comme une réalité extérieure, mais comme une manifestation de son être le plus profond. Elle est, si paradoxale que cela puisse paraître, la véritable liberté, parce qu'elle exprime ce qu'il y a de plus profond dans l'homme, ce qui lui permet de résister au monde qui l'accable, c'est-àdire la conscience, l'honneur pour reprendre un mot de Péguy, de Bernanos et de Vigny. Camus écrit: "On ne saurait aimer vraiment les autres si l'on ne s'estime pas d'abord. Non au plus haut, mais au juste prix. Et quel est le prix de l'homme qui bouche ses oreilles au cri de la victime et qui, devant l' injustice, consent à baisser le front"?

Au-delà donc des termes du conflit entre le devoir et le bonheur, une force irrésistible et illogique le résout. Mais efface-t-elle l'exigence du bonheur? Elle l'intègre plutôt à l'intérieur d'un cercle plus large qui enserre toute l'humanité souffrante. Le bonheur particulier s'anéantit devant le bo- 
nheur de tous: mais en s'anéantissant, il renaît plus pur et plus grand, car c'est la même lumière qui l'éclaire, celle qui illuminait l'appétit de vivre de Camus adolescent et qu'il n'a jamais ni oubliée, ni reniée.

Dans cet élargissement de la morale individuelle à la morale personnelle, qui est celle de la fraternité, la recherche du bonheur n'est donc pas abandonnée; elle prend au contraire tout son sens, celui d'un long cheminement vers des valeurs plus hautes.

Que nous voilà loin de l'hédonisme de Voltaire auquel certains, comme P. H. Simon, voudraient ramener la philosophie du bonheur de Camus! Il rejette cette molle complaisance au mal et cette satisfaction facile dont se contentait le patriarche de Ferney. "Il y a beaucoup d'hommes heureux, disait Voltaire. Il en est des hommes comme des animaux, tel chien couche et mange avec sa maîtresse; tel autre tourne la broche et est tout aussi content".

Elle suppose une tension farouche de tout l'être vers les sommets, une volonté d'affirmer, envers et contre tout, la dignité de l'homme. Elle est inséparable chez lui du salut intérieur et de la recherche des valeurs les plus nobles". Qu'estce que sauver l'homme?... Je vous le crie de tout moi-même, c'est ne pas le mutiler et c'est donner ses chances à la justice qu'il est seul à concevoir" (Lettres à un ami allemand). Et ailleurs, dans le même texte: "A travers les clameurs et la violence, nous tentions de garder au coeur le souvenir d'une mer heureuse, d'une colline jamais oubliée, le sourire d'un cher visage". Le bonheur conserve, même lorsqu'il parait se sacrifier à l'exigence de justice, la lumière transparente de la beauté et de l'amour.

\section{CONCLUSION}

\section{La "fidélité quotidienne"}

Nous voilà au terme d'un itinéraire qui a conduit Camus, par une marche où l'unité intérieure de sa conscience est restée intacte, de la joie de vivre jusqu'à la fraternité humaine. En effet, ni "La Chute", ni "L'Exil et le Royaume" n'apportent rien de vraiment nouveau à son univers intérieur. De ces deux 
livres, il disait à un ami italien (Giacomo Antonini) qu'il s'agissait d'intermèdes avant la prochaine étape, qui serait la plus importante et qui commencerait par un roman, celuilà même que Camus préparait avant sa mort. Quelle aurait été cette étape? Contentons-nous de l'enrichissement de celles que nous connaissons et des enseignements qu'elles nous apportent. Le premier est la fidélité à soi-même et le refus des simplifications faciles.

Sur cet itinéraire, Camus, comme il l'a écrit, après un retour à Tipasa, qui a été aussi l'occasion d'un retour sur sa jeunesse, n'a rien oublié de lui-même: “Je n'ai pu renier la "lumière où je suis né, et cependant je n'ai pas voulu refuser "les servitudes de ce temps. Il y a pour les hommes d'aujourd" "hui un chemin intérieur que je connais bien pour l'avoir "parcouru dans les deux sens et qui va des collines de l'esprit "aux capitales du crime... Oui, il y a la beauté et il y a les "humiliés. Quelles que soient les difficultés de l'enteprise, je "voudrais n'être jamais infidèle ni à l'une, ni aux autres".

Pour Camus en effet, la conscience doit maintenir jusqu' au bout à la fois la volonté de bonheur et la nécessité du sacrifice: c'est dans cette tension lucide que l'homme se retrouve pleinement et atteint à ses sommets. Parlant d'un héros de la Résistance mort au combat, il écrit: "Bien entendu, il y a dans tout sacrifice du hasard... Pourtant la différence est grande entre ceux qui choisissent de risques et ceux qui choisissent de se taire. Et parmi ceux qui risquent, entre ceux qui le font jusqu'au bout et ceux qui renoncent; et parmi ceux qui vont jusqu'à la consommation, entre les uns qui n'ont aucun motif de vivre et les autres qui, face aux plus hautes raisons de durer, entretiennent jusqu'à la fin, la conscience déchirée $\mathrm{du}$ bonheur auquel ils renoncent et du devoir qui va les tuer. Ceux-là, et eux seuls, ont su racheter, jour après jour, le déshonneur où nous nous survivons".

Ce serment de fidélité, Camus l'a tenu jusqu'au bout, jusque dans son silence de ces deux dernières années au sujet da la tragédie algérienne, qui était peuplé d'interventions discrètes et efficaces contre toutes les injustices qu'engendre cette guerre affreuse. Il l'a tenu aussi dans ses oeuvres qui allient la générosité et la beauté; il l'a tenu enfin dans sa 
vie quotidienne, dans l'effort patient et modeste qu'elle a constitué pour apporter plus de justice et plus d'humanité à un monde torturé. La recherche du bonheur ne s'égare pas chez lui sur des chemins où l'héroïsme n'est que le masque de l'orgueil et de la violence; elle n'aboutit pas non plus à une justification du plaisir égoïste et désespérant; elle est une tentative sans cesse renouvelée pour que le corps, la tendresse, la création de la noblesse humaine reprennent leur place dans ce monde insensé. "Il y a des dieux de lumière et des idoles de boue. Mais c'est le chemin moyen qui mène aux visages de l'homme qu'il s'agit de trouver".

Ce n'est pas un hasard si le héros de "La Peste" est un médecin: "Je m'intéresse, dit Rieux, à la santé de l'homme". Et plus loin: Il ne s'agit d'honnêteté - Qu'est-ce que l'hon"nêteté, dit Rambert, d'un air soudain sérieux? - Je ne sais "pas ce qu'elle est en général, reprend Rieux. Mais dans mon "cas, je crois qu'elle consiste à faire son métier".

Les "vrais médecins", comme Rieux, les "saints sans Dieu", idéal de Tarrou, sont des hommes qui ne sont ni victimes ni bourreaux qui se refusent à admettre la souffrance et qui la soignent à leur place de toutes leurs forces. Ce sont des hommes qui veulent guérir, de toutes leurs forces, dans la mesure du possible, les enfants torturés, dont le sort incompréhensible dans son injustice totale, révolte Rieux et oppose un obstacle invincible à son amour pour la création. S'adressant aux chrétiens, dans un exposé qu'il fit au couvent des Dominicains de Latour-Maubourg, Camus s'écriait: "Nous "ne pouvons pas empêcher peut-être que cette création soit "celle où des enfants sont torturés. Mais nous pouvons dimi"nuer le nombre d'enfants torturés. Et si vous ne nous y aidez "pas, qui donc, dans le monde, pourra nous y aider?"

Cette modestie apparente, cette tâche limitée fixée à l' action humaine, sont, à vrai dire, une grande sagesse et une garantie d'efficacité. "Sa pensée de midi", qui est l'aboutissement de la longue méditation sur le destin de l'homme que constitue "L'Homme Révolté" rassemble cette leçon et lui donne toute sa valeur. Le bonheur que Camus ne se dissocie pas de la volonté de justice, est bien loin de toute illusion et de tout enivrement, même s'il conserve encore le reflet de 
l'illumination et de l'éblouissement des jours de son adolescence. De tout son amour, de toute sa volonté, de toute son intelligence, il se voue, pour se mettre au serviçe de l'homme, à la "fidélité quotidienne". C'est grâce à son effort renouvelé que, comme Sisyphe, l'homme atteindra les sommets. Ecoutons résonner, dans les dernières lignes de "L'Homme Révolté", cette voix discrète et puissante, plus puissante, plus convaincante et plus émouvante que tant de déclarations vides et fracassantes:

"O cette heure où chacun de nous doit tendre l'arc pour "refaire ses preuves, conquérir, dans et contre l'histoire, ce "qu'il possède déjà, la maigre moisson de ses champs, le bref "amour de cette terre, à l'heure où naît enfin un homme, il "faut laisser l'époque et ses fureurs adolescentes. L'arc se tord, "le bois crie. Au sommet de la plus haute tension va jaillir "l'élan d'une droite flèche, du trait le plus dur et le plus "libre". 